\title{
Percepción de acoso o discriminación durante la residencia de urología en Colombia
}

\section{Perception of Harrasment or Discrimination during Urology Residency in Colombia}

\author{
Ana Maria Ortiz-Zableh ${ }^{10}$ Andres Felipe Quiñones Roa ${ }^{1}$ Bernardo Santamaria Fuerte ${ }^{1,2}$ \\ Veronica Tobar-Roa ${ }^{1,2}$
} ${ }^{1}$ Departamento de Urología, Universidad Autónoma de Bucaramanga
(UNAB), Floridablanca, Santander, Colombia
2 Centro Urológico Foscal, Clínica FOSCAL, Floridablanca, Santander,
Colombia
Dirección para correspondencia Ana Maria Ortiz-Zableh, MD, Departmento de Urología, Universidad Autónoma de Bucaramanga (UNAB), Calle 155A \# 23-60, Floridablanca 681004, Santander, Colombia (e-mail: Aortiz779@unab.edu.co).

Urol Colomb 2021;30(3):e179-e183.

\section{Resumen}

\section{Palabras Clave}

- acoso escolar

- internado y residencia

- urología

- sexismo

- Colombia
Objetivos El maltrato laboral (acoso, abuso o discriminación) ejercido de manera persistente contra un individuo genera un ambiente de trabajo hostil y lleva a agotamiento, con un impacto psicológico importante y la posibilidad de aparición de síntomas depresivos. En Colombia, no contamos con publicaciones al respecto. El objetivo de nuestro trabajo es conocer la percepción de los residentes de urología en cuanto a discriminación de género y acoso laboral, verbal y sexual durante su formación.

Metodos Estudio observacional, descriptivo, de corte transversal, por medio de encuestas anónimas. Evaluamos las características demográficas, la percepción de acoso, el tipo de acoso, si éste afectó el rendimiento laboral del residente, y por parte de quién lo percibió.

Resultados Obtuvimos respuestas de $82 / 115(71,3 \%)$ residentes, en su mayoría hombres (45 [56\%]). En total, 66\% (54) reportaron haber experimentado acoso laboral; y 35,4\% (29) reportaron haber experimentado discriminación de género, siendo más frecuente en las mujeres $(17 ; 58,6 \%)$. El acoso verbal fue reportado por un $64,6 \%$ (53), y afectó el trabajo de un 92,5\%. La percepción de acoso laboral fue similar entre hombres y mujeres (32 [69.5\%] hombres y 21 [61.1\%] de mujeres). En total, 7 $(19 \%)$ mujeres reportaron acoso sexual. Con respecto a la fuente de acoso, $39(65,8 \%)$ fue por profesores, 26 (45\%), por otros residentes, y 17 (35.4\%), por pacientes.

Conclusión La percepción de acoso durante la residencia de urología en Colombia es real, y afecta el trabajo de los residentes. Este acoso es mayor por parte de los hombres, y es principalmente generado por profesores. Consideramos nuestro trabajo el punto de partida para continuar investigando un tema de importancia a nivel nacional y internacional. recibido

10 de enero de 2021

aceptado

02 de mes de julio de 2021
DOI https://doi.org/

$10.1055 / \mathrm{s}-0041-1737012$

ISSN 0120-789X.

e ISSN 2027-0119.
(C) 2021. Sociedad Colombiana de Urología. All rights reserved. This is an open access article published by Thieme under the terms of the Creative Commons Attribution-NonDerivative-NonCommercial-License, permitting copying and reproduction so long as the original work is given appropriate credit. Contents may not be used for commercial purposes, or adapted, remixed, transformed or built upon. (https://creativecommons.org/ licenses/by-nc-nd/4.0/)

Thieme Revinter Publicações Ltda., Rua do Matoso 170, Rio de Janeiro, RJ, CEP 20270-135, Brazil 


\begin{abstract}
Keywords

- harassment

- internship and residency

- urology

- sexism

- Colombia

Purpose Workplace bullying (harassment, abuse, or discrimination), practiced persistently against an individual, can generate a hostile workplace environment, consequently leading to exhaustion, with poor psychological outcomes, and the onset of symptoms such as loss of confidence, fatigue, depressive thoughts, desertion, and suicidal thoughts. There are no publications regarding this issue in Colombia.

Our objective is to describe the residents' perception of gender and workplace discrimination and verbal/sexual harassment during their urological training.

Methods Observational, descriptive, cross-sectional study with anonymous surveys. We evaluated the demographic characteristics and the residents' perception of harassment, the type of harassment, how it affected their performance during residency, and from whom it was perceived.

Results We were able to obtain answers from 82/115 residents (71.3\%), most of them men (45 [56\%]). In total, 66\% (54) reported workplace harassment; and 35.4\% (29) felt gender discrimination, most of them women $(17 ; 58.6 \%)$. Verbal abuse was reported by $64.6 \%$ (53), and it affected the work of $92.5 \%$. The paerception of workplace harassment was similar among both men and women (32 [69.5\%] men and 21 [61.1\%] women). A total of $7(19 \%)$ women reported sexual abuse. Regarding the source of the abuse, 39 (65.8\%) was by professors, 26 (45\%), by other residents, and 17 (35.4\%), by patients.

Conclusion The perception of harassment during urology residency in Colombia is real, and it affects the work of residents. This abuse is greater on the part of men, and is mainly perpetrated by professors. We consider our work the starting point to continue researching a topic of national and international importance.
\end{abstract}

\section{Introducción}

El maltrato laboral, entendido como acoso, comportamiento intimidatorio, abuso o discriminación que se ejerce de manera persistente contra un individuo, puede generar un ambiente de trabajo hostil y consecuentemente llevar a agotamiento, con un impacto psicológico importante y la aparición de síntomas como pérdida de confianza, fatiga, sentimientos depresivos y deserción, pudiendo generar incluso ideas suicidas. ${ }^{1,2}$ Estos comportamientos en muchos casos no son identificados, e incluso son tolerados y justificados, considerándose inherentes a la formación médica.

Dentro de las especialidades médico-quirúrgicas, la urología es una de las que reporta una mayor incidencia de síndrome de agotamiento, ${ }^{3}$ aunque es llamativo que el tema de acoso propiamente dicho no haya sido estudiado a fondo. Hasta el momento, Colombia no cuenta con publicaciones sobre la percepción de acoso, abuso o discriminación durante la residencia de urología.

Nuestro objetivo es conocer la percepción de los residentes de urología de Colombia con respecto a la discriminación de género y al acoso laboral, verbal y sexual durante los años de formación.

\section{Metodos}

Realizamos un estudio observacional, descriptivo, de corte transversal, aplicando una encuesta anónima desarrollada por los autores, y con permiso de la Sociedad Colombiana de Urología (SCU), enviada por medio de una plataforma electrónica de la SCU a los residentes que aceptaron participar.

A todos los residentes se les invitó a diligenciar la encuesta, pudiendo aceptar o rechazar dicha invitación. Se respetaron los principios éticos fundamentales mediante un consentimiento verbal que se reflejó en el diligenciamiento autónomo y voluntario del instrumento de recolección.

Se evaluaron las características demográficas, la percepción de acoso, el tipo de acoso, si éste afectó su rendimiento durante la residencia, y por parte de quién lo percibió (- Apéndice A). Se calculó una muestra no probabilística de los participantes que aceptaron responder la encuesta. Se realizó un análisis descriptivo en el programa Stata (StataCorp., LLC, Colege Station, TX, EEUU), versión 15. El componente descriptivo se elaboró según la naturaleza de las variables; las cualitativas, mediante frecuencias absolutas y relativas, y las cuantitativas, con medidas de tendencia central, posición y dispersión según la distribución de frecuencias. Se estimó la diferencia por sexo en la percepción de acoso por otros residentes, profesores, personal de enfermería, y pacientes con una prueba binomial para contrastar dos proporciones. El nivel de significancia del estudio fue del $5 \%$.

\section{Resultados}

Se invitó a participar a 115 residentes de urología de Colombia en febrero de 2020; del total, 62 (54\%) eran 
Tabla 1 Características demográficas de los residentes encuestados

\begin{tabular}{|l|l|l|}
\hline $\mathbf{N}$ & \multicolumn{3}{|l|}{$\mathbf{8 2}$} \\
\hline \multirow{3}{*}{$\begin{array}{l}\text { Género: }(\%) \\
\end{array}$} & Masculino & $46(56 \%)$ \\
\cline { 2 - 3 } & Femenino & $36(43,9 \%)$ \\
\cline { 2 - 3 } & Diverso & 0 \\
\hline \multirow{3}{*}{$\begin{array}{l}\text { Año de residencia: } \\
\end{array}$} & Primero & $17(20,7 \%)$ \\
\cline { 2 - 3 } & Segundo & $27(32,9 \%)$ \\
\cline { 2 - 3 } & Tercero & $20(24,3 \%)$ \\
\cline { 2 - 3 } & Cuarto & $17(20,7 \%)$ \\
\hline \multirow{2}{*}{$\begin{array}{l}\text { Estado civil: } \\
\mathbf{n}(\%)\end{array}$} & Soltero & $71(86,5 \%)$ \\
\cline { 2 - 3 } & Casado & $5(6,09 \%)$ \\
\cline { 2 - 3 } & Unión libre & $6(7,3 \%)$ \\
\hline
\end{tabular}

hombres, y 53 (46\%), mujeres (datos suministrados por la SCU en febrero de 2020). De los 115 urólogos en formación, se lograron obtener las respuestas de las encuestas de 82 (71,3\%) (-Tabla 1 ).

Con respecto a la percepción de acoso laboral, 66\% ( $n=54)$ de los encuestados reportó haber experimentado agresión/hostigamiento laboral durante su residencia; considerando las respuestas no excluyentes, se encontró que este tipo de acoso generó una afectación en sus labores en un $89 \%$ (48) de ellos. Con respecto a la discriminación de género, el 35,4\% (29) reportó haberla presentado, y 16 (55,2\%) de ellos consideraron que estas conductas afectaron sus labores como residentes. Sobre el acoso verbal, el 64,6\% (53) refirió haber sido objeto de insultos o críticas consideradas destructivas, viéndose afectado su trabajo como residente en 49 de ellos (92,5\%).

La percepción de acoso laboral fue similar entre hombres y mujeres (69,5\% y 61,1\%, respectivamente). En cuanto a la discriminación de género, el porcentaje fue significativamente superior en las mujeres encuestadas (7 [26\%] de los hombres y 13 [47.2\%] de las mujeres). Con respecto al acoso sexual, éste ha sido percibido por 7 (19\%) mujeres; todas las que respondieron afirmativamente refirieron que esto afectó su trabajo y su desarrollo como residentes.

Finalmente, se hizo una caracterización de la fuente del acoso (pacientes, profesores, residentes, personal de enfermería, o ninguno de los anteriores). En total, 6 (10\%) encuestados respondieron ninguno de los anteriores; del $90 \%$ restante, el 45\% se sintió acosado por otros residentes (37: 26 hombres y 11 mujeres; $p=0,0112$ ), 65,8\% se sintió acosado por profesores (54: 31 hombres y 23 mujeres; $p=0,740$ ), $29 \%$, por personal de enfermería (24: 13 hombres y 11 mujeres; $p=0,821), y 35,4 \%$, por pacientes (29: 15 hombres y 14 mujeres; $p=0,555$ ).

\section{Discusión}

Los resultados de nuestra encuesta muestran que $66 \%$ de los residentes han percibido acoso laboral, y 35,4\%, discriminación de género (siendo 7 (26\%) hombres y 13 (47.2\%) mujeres).

El acoso o maltrato puede llevar a una disminución en la satisfacción percibida por el residente acerca de su proceso de formación, y consecuentemente llevar a un maltrato secundario hacia los pacientes. ${ }^{1}$ Es un problema que no sólamente se ha estudiado en Estados Unidos, sino en Asia, Canadá, y Europa, ${ }^{1,2,4}$ aunque existe poca literatura publicada al respecto a nivel mundial. En una revisión sistemática y metanálisis de 59 artículos realizada en 2011, Fnais et al. $^{3}$ encontraron que $59,4 \%$ de los médicos en entrenamiento habían experimentado algún tipo de acoso o discriminación (intervalo de confianza del 95\% [IC95\%]: $52,0-66,7 \%$ ), siendo el abuso verbal el más frecuentemente reportado. El acoso fue mayormente percibido por parte de los especialistas, seguido de los pacientes y los familiares de los pacientes. ${ }^{3}$

Fnais et al. $^{5}$ aplicaron una encuesta a 213 residentes en Arabia Saudita en 2013, y encontraron que 83,6\% había percibido algún tipo de acoso, siendo más común el verbal (61,5\%), seguido de la discriminación de género (58,3\%). El abuso sexual fue reportado por $19,3 \%$ de los residentes, la mayoría de éstos mujeres $(p=0,061) .^{5}$ Nuestra encuesta muestra que los residentes habían sentido acoso principalmente por parte de profesores (tanto los hombres como las mujeres), seguido por otros residentes (39 (65.8\%) profesores y 26 (45\%) otros residentes).

El acoso laboral es un problema que se ha relacionado con las especialidades quirúrgicas. En un estudio ${ }^{4}$ reciente publicado en el New England Journal of Medicine, se realizó una encuesta a residentes de especialidades quirúrgicas en Estados Unidos: 31,9\% de los participantes (65\% de mujeres) reportaron discriminación de género; $30.2 \%$ refirieron abuso verbal o emocional (33\% de mujeres), siendo la mayoría de las veces por un cirujano tratante $(52,4 \%)$, y otros residentes (20\%). El abuso físico sólo fue reportado por un $2,2 \%$. El abuso sexual fue reportado por $10.3 \%$ de los encuestados, siendo principalmente por pacientes o familiares de pacientes $(31,2 \%)$, cirujanos $(30,9 \%)$, residentes $(15,4 \%)$, y personal de enfermería $(11,7 \%){ }^{4}$

Fitzgerald et al. ${ }^{6}$ aplicaron la herramienta de tamizaje HITS (Hurt, Insulted, Threatened with harm or Screamed at, en inglés), en un estudio multicéntrico a 76 residentes de cirugía, y encontraron que el abuso sexual eral el más frecuente (28\%), seguido de la discriminación por género (15,7\%), y la discriminación por raza (7,9\%). Además, se les preguntó a los participantes acerca del impacto emocional que traía consigo el acoso; $36,8 \%$ respondió que sentía rabia, $31,6 \%$, frustración, 30,2\% estaban avergonzados, y $25 \%$ reportó ansiedad. ${ }^{6}$ En nuestro estudio, de los residentes que sintieron acoso laboral, 48 de 54 (88\%) respondieronque este acoso había afectado su trabajo como residente.

Llama la atención que, a pesar de que el número de mujeres en la residencia de urología a nivel nacional ha aumentado drásticamente en los últimos años, ${ }^{7}$ y de que el número de mujeres en cargos académicos y ejecutivos es cada vez mayor, persista la percepción de discriminación de 
género y acoso sexual. ${ }^{8}$ Como no existen estudios previos al respecto, desconocemos si este acoso era mayor previamente, cuando habían menores mujeres en la especialidad.

Un metanálisis ${ }^{9}$ de 2014 encontró una percepción de acoso sexual con una media de prevalencia del 33\% en estudiantes y residentes mujeres, comparada con $4 \%$ de los hombres. Ortiz Zableh et al. ${ }^{7}$ aplicaron encuestas a urólogas y residentes mujeres de urología en Colombia, y encontraron que 18 de 53 urólogas (34\%), y 15 de 36 residentes $(41,7 \%)$ reportaban acoso laboral por el hecho de ser mujer. Nuestra encuesta demostró que 7 (19\%) residentes habían percibido acoso sexual durante la residencia, todas mujeres.

Nuestro estudio tiene ciertas desventajas, como el hecho de haber sido aplicado de forma virtual, sin contar con una representación total de los residentes a nivel nacional. Es el primer trabajo a nivel nacional que evalúa la percepción de acoso por parte de los residentes de urología, y demuestra cómo se afecta la calidad del trabajo en la residencia en urología por el acoso. Se requieren de más estudios a nivel nacional e internacional, que incluyan residentes de otras especialidades, para sacar más resultados y evaluar las posibles razones detrás del acoso (sobrecarga laboral, discriminación de género, discriminación por parte de pacientes, entre otras).

\section{Conclusión}

La percepción de acoso durante la residencia de urología en Colombia es real y afecta el trabajo de los residentes. Este acoso es mayor por parte de los hombres, es principalmente generado por profesores y otros residentes, y afecta la calidad del trabajo durante su formación como especialistas. Consideramos nuestro trabajo el punto de partida para continuar investigando un tema de importancia a nivel nacional y internacional.

Financiamiento

Los autores no tienen fuente de financiación que declarar.

Conflicto de Intereses

Los autores no tienen conflicto de intereses que declarar.

\section{Referencias}

1 Nagata-Kobayashi S, Maeno T, Yoshizu M, Shimbo T. Universal problems during residency: abuse and harassment. Med Educ 2009;43(07):628-636

2 Rodríguez-Socarrás M, Vasquez JL, Uvin P, Skjold-Kingo P, Gómez Rivas J. "Síndrome de agotamiento": Estrés, Burnout y depresión en Urología. Arch Esp Urol 2018;71(01):46-54

3 Fnais N, Soobiah C, Chen MH, et al. Harassment and discrimination in medical training: a systematic review and meta-analysis. Acad Med 2014;89(05):817-827

$4 \mathrm{Hu}$ Y-Y, Ellis RJ, Hewitt DB, et al. Discrimination, Abuse, Harassment, and Burnout in Surgical Residency Training. N Engl J Med 2019;381(18):1741-1752

5 Fnais N, al-Nasser M, Zamakhshary $M$, et al. Prevalence of harassment and discrimination among residents in three training hospitals in Saudi Arabia. Ann Saudi Med 2013;33(02):134-139

6 Fitzgerald CA, Smith RN, Luo-Owen X, et al. Screening for harassment, abuse, and discrimination among surgery residents: An EAST multicenter trial. Am Surg 2019;85(05):456-461

7 Ortiz Zableh AM, Santamaría Fuerte B, Tobar Roa V. Situación actual de la mujer en Urología en Colombia. Arch Esp Urol 2021;74 (02):187-196

8 Bates CK, Jagsi R, Gordon LK, et al. It Is Time for Zero Tolerance for Sexual Harassment in Academic Medicine. Acad Med 2018;93 (02):163-165

9 Jagsi R, Griffith KA, Jones R, Perumalswami CR, Ubel P, Stewart A. Sexual harassment and discrimination experiences of academic medical faculty. JAMA 2016;315(19):2120-2121 


\section{Apéndice A Preguntas aplicadas}
1. Sexo
a. Hombre
b. Mujer
c. Diverso

2. Año de residencia
a. 1
b. 2
c. 3
d. 4

3. Estado civil
a. Soltero
b. Casado
c. Unión libre
d. Viudo

4. ¿Ha sentido acoso laboral durante la residencia?
a. Sí
b. No

5. ¿Si su respuesta a la pregunta 4 fue sí, esta percepción de acoso ha hecho que su trabajo como residente se vea afectado? a. Sí

b. No

6. ¿Ha sentido discriminación de género durante la residencia?
a. Sí
b. No

7. ¿Si su respuesta a la pregunta 6 fue sí, esta percepción de acoso ha hecho que su trabajo como residente se vea afectado?
a. Sí
b. No

8. ¿Ha sentido acoso verbal durante la residencia?
a. Sí

b. No

9. ¿Si su respuesta a la pregunta 8 fue sí, esta percepción de acoso ha hecho que su trabajo como residente se vea afectado? a. Sí

b. No

10. ¿Ha sentido acoso sexual durante la residencia?
a. Sí
b. No

11. ¿Si su respuesta a la pregunta 10 fue sí, esta percepción de acoso ha hecho que su trabajo como residente se vea afectado? a. Sí

b. No

12. ¿Por parte de quiénes ha percibido acoso? (marque cualquiera de las opciones)
a. Residentes
b. Profesores
c. Personal de enfermería
d. Pacientes
e. Otros (especifique) 